\title{
The effects of kinesiotaping on wrist extensor strength using an isokinetic device in patients with chronic lateral epicondylitis: A randomized-controlled trial
}

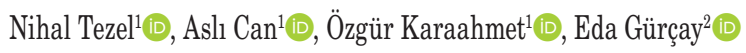 \\ ${ }^{1}$ Department of Physical Medicine and Rehabilitation, Ankara Ylldırım Beyazıt Training and Research Hospital, Ankara, Turkey \\ ${ }^{2}$ Department of Physical Medicine and Rehabilitation, Ankara Gaziler Physical Therapy and Research Hospital, Ankara, Turkey
}

Received: June 25, 2018 Accepted: November 28, 2019 Published online: March 03, 2020

\begin{abstract}
Objectives: This study aims to evaluate short-term effects of kinesiotaping (KT) on pain, arm function, grip strength, and wrist extensor strength in patients with chronic lateral epicondylitis (LE).

Patients and methods: A total of 48 patients (32 females, 16 males years; mean age 47.6 years; range 27 to 67 years) with chronic LE were randomly assigned to either KT group $(n=27)$ or sham group $(n=21)$. Pain intensity with visual analog sclae (VAS), arm pain and function with Patient-Rated Tennis Elbow Evaluation Questionnaire (PRTEE), grip strength with hand dynamometer, and wrist extensor strength by an isokinetic device were evaluated before and at the end of the treatment. The KT stayed on for five days and the procedure was repeated for three times.

Results: Although pain and functional levels of patients with chronic LE were significantly improved both with KT (pain, $\mathrm{p}=0.001$; function, $\mathrm{p}=0.001$ ) and sham groups (pain, $\mathrm{p}=0.001$; function, $\mathrm{p}=0.001$ ), no significant difference was observed between the groups.

Conclusion: Both KT and sham taping provided similar improvement in pain relief through arm functions in patients with chronic LE.

Keywords: Function, kinesiotape, lateral epicondylitis, pain.
\end{abstract}

Lateral epicondylitis (LE), also known as tennis elbow, is a common pathology of the elbow, affecting 1 to $3 \%$ of middle-aged individuals in general population. ${ }^{[1-3]}$ It is usually attributed to the extensor carpi radialis brevis. ${ }^{[4]}$ Although its exact pathogenesis still remains unknown, the most accepted theory is that LE is a result of inflammatory responses of the soft tissue due to the microscopic laceration of the extensor common tendon attached to the lateral epicondyle. ${ }^{[5]}$ Until now, several treatments have been suggested for LE; however, there is no consensus on the optimal treatment. Nonetheless, some general principles can be taken into consideration. The treatment of LE is usually orientated to the management of pain, preservation of movement, improvement in grip strength, return to normal function, and control of further clinical deterioration. ${ }^{[6]}$

In recent years, it has been proposed to use kinesiotaping $(\mathrm{KT})$ as a non-invasive treatment method to restore the normal function of the muscles and joints, to reduce pain, to maintain normal biomechanics of the tissue, and to restore tissue hemostasis in the field of rehabilitation. This method can be used as a dependent treatment approach or as a means to maintain treatment effects of other methods. ${ }^{[7]}$

The KT was first developed by Dr. Kenzo Kase, a practitioner licensed in chiropractic and acupuncture, in 1973 in Japan. The KT fibers are designed to stretch longitudinally up to $40 \%$ of it resting length compared to previous tapes. ${ }^{[8]}$ Its exact mechanism has not been 
clearly understood, yet. However, one of the proposed mechanisms for the use of $\mathrm{KT}$ is that it decreases the pressure on muscles and soft tissues, affecting the cutaneous mechanoreceptors, which leads to tensional force and mechanical pressure on the skin. Kinesio tape is an elastic tape that allows the full range of motion of a joint. ${ }^{[7-9]}$

Although there are many studies about taping techniques in the conservative treatment of $\mathrm{LE},{ }^{[10-12]}$ there is a very limited number of data about KT. ${ }^{[13-16]}$ Most of the studies in the literature have examined non-elastic tape effects. ${ }^{[10-12]}$ In the present study, we, therefore, aimed to evaluate short-term effects of $\mathrm{KT}$ on pain, arm function, grip strength, and wrist extensor strength in patients with chronic LE.

\section{PATIENTS AND METHODS}

This randomized-controlled study was conducted at University of Health Sciences, Dışkapı Yıldırım Beyazıt Training and Research Hospital, Physical Therapy and Rehabilitation Clinic between November 2017 and May 2018. A total of 72 patients with LE were initially included in this study. Inclusion criteria were as follows: age between 18 to 65 years; pain and tenderness on the lateral epicondyle for at least three months; and provocation of the lateral elbow pain with at least one of the tests (i.e., resisted wrist extension, resisted middle finger extension, or passive stretch of wrist extensors). Exclusion criteria were as follows: history of upper extremity trauma/fracture, cervical discopathy and/or narrow cervical spinal canal, neuropathy or arthritis in the upper extremities, physical therapy or injection therapy for LE within the last three months and pregnancy. Of 72 patients, a total of 48 ( 32 females, 16 males; mean age 47.6 years; range 27 to 67 years) completed the study. These patients were randomly assigned to either KT group $(n=27)$ or sham group $(n=21)$. The study flow chart is shown in Figure 1.
A written informed consent was obtained from each patient. The study protocol was approved by the University of Health Sciences, Dışkapı Yıldırım Beyazıt Training and Research Hospital Ethics Committee. The study was conducted in accordance with the principles of the Declaration of Helsinki.

\section{Intervention}

A standard 2-inch $(5 \mathrm{~cm}) \mathrm{Kinesio}^{\circ} \mathrm{Tex}$ tape (Kinesio Holding Corporation, Albuquerque, New Mexico, USA) was used in both groups. It was applied to the forearm in a sitting position by a certified KT researcher. The KT stayed on for five days and the procedure was repeated for three times.

In the KT group, muscle inhibition and fascia correction techniques were used. With the muscle inhibition technique, we applied a long Y-shaped strip to the wrist extensors from insertion to origin after stretching the muscle with 15 to $25 \%$ tension. We used this technique to inhibit the overused muscles function. ${ }^{[17]}$ With the fascia correction technique, 25 to $35 \%$ tension was applied by a short Y-shaped strip to support the fascia (Figure 2). ${ }^{[18]}$

In the sham group, the technique was performed with 2 I-shaped strips without any tension of the skin. A long I strip was applied to the wrist extensor muscles and a short I strip was applied horizontally just below the lateral epicondyle (Figure 3). ${ }^{[19]}$

\section{Outcome measures}

Demographic characteristics of the patients were recorded. Resisted wrist extension, resisted middle finger extension, and passive stretch of wrist extensors test results were evaluated. Pain on the lateral epicondyle was accepted as positive for each test. Lateral epicondyle pain during the last week was evaluated using the visual analog scale (VAS) $(0-10 \mathrm{~cm})$. Tenderness over the lateral epicondyle was graded from 0 to 3 ( $0=$ none, $1=$ mild, $2=$ moderate, 3 =severe).

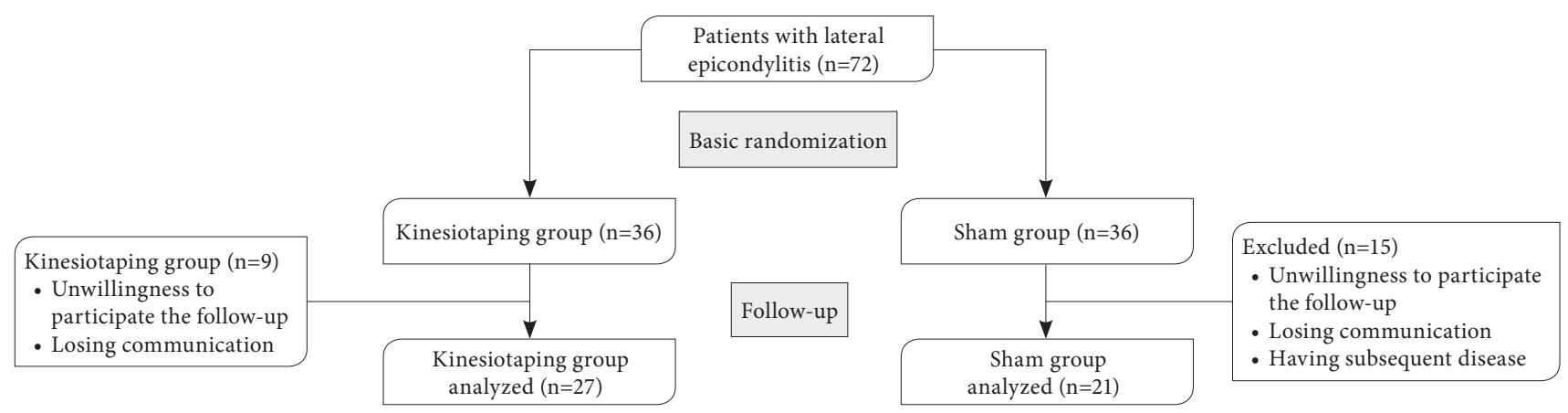

Figure 1. Study flow chart. 


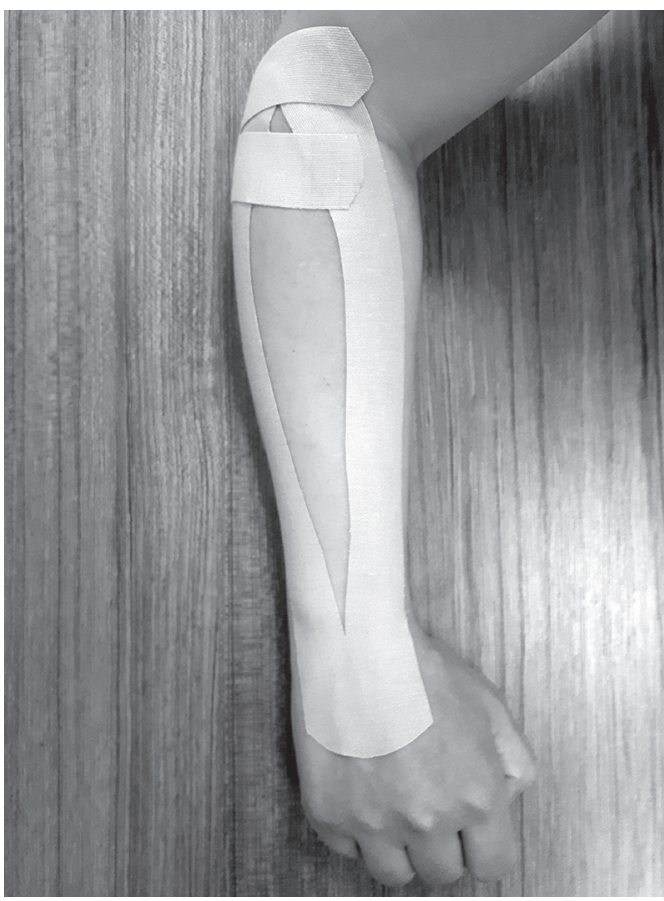

Figure 2. Kinesiotaping application.

The Patient-Rated Tennis Elbow Evaluation Questionnaire (PRTEE) was used to evaluate pain and disability of the patients with LE. It is a highly specific, 15-item self-report questionnaire for LE consisting of two subscales: pain subscale and function subscale. Pain subscale has five items and function subscale has 10 items. Each item is scored on a $0-10$ scale $(0=$ no pain or no difficulty, $10=$ worst ever or unable to do).

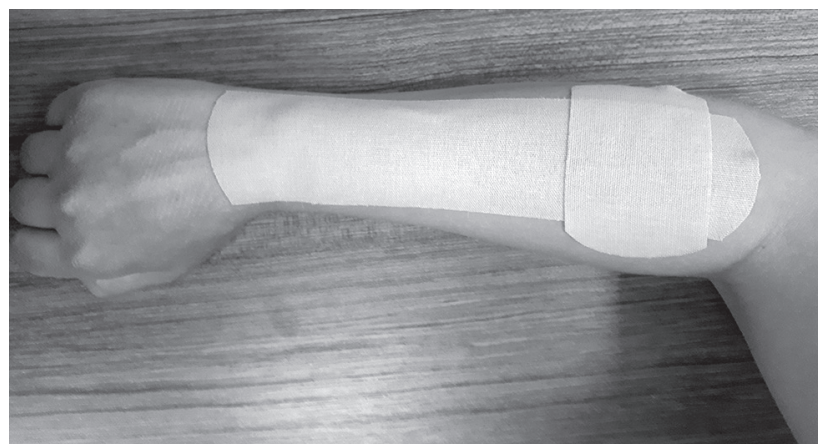

Figure 3. Sham taping application.

Each subscale score ranges from 0 to 50 , and the total score ranges from 0 to 100 , where higher scores indicate greater pain and disability. Its validation and reliability studies have been conducted in the Turkish population by Altan et al. ${ }^{[20]}$

The grip strength of the affected side was measured using a Jamar hydraulic hand dynamometer (Sammons Preston Inc., Bolingbrook, IL, USA). All measurements were done in the sitting position, elbow at $90^{\circ}$ flexion, and the wrist in a neutral. The patients were asked to hold the dynamometer and grip the handle of the dynamometer as hard as possible for three times. The average values of three trials were recorded in kilogram.

The wrist extensor strength was measured by an isokinetic dynamometer Biodex System 3

\begin{tabular}{|c|c|c|c|c|c|c|c|c|c|c|c|}
\hline & & & Demograph & and cli & $\begin{array}{l}\text { TABLE } 1 \\
\text { cal charac }\end{array}$ & istic & of th & patients & & & \\
\hline & & & nesiotaping & oup (n= & & & & Sham grol & $(\mathrm{n}=21)$ & & \\
\hline & $\mathrm{n}$ & $\%$ & Mean \pm SD & Mean & Min-Max & $\mathrm{n}$ & $\%$ & Mean \pm SD & Mean & Min-Max & $p$ \\
\hline Age (year) & & & $48.4 \pm 10.6$ & & & & & $46.8 \pm 5.1$ & & & 0.52 \\
\hline Gender & & & & & & & & & & & \\
\hline Male & 9 & 33.3 & & & & 7 & 33.3 & & & & \\
\hline Female & 18 & 66.7 & & & & 14 & 66.7 & & & & \\
\hline Education (years) & & & & 8 & $5-15$ & & & & 8 & $0-11$ & 0.73 \\
\hline Occupation & & & & & & & & & & & 0.81 \\
\hline Housewife & 7 & 25.9 & & & & 8 & 38.1 & & & & \\
\hline Worker & 11 & 40.2 & & & & 8 & 37.6 & & & & \\
\hline Retired & 9 & 33.9 & & & & 5 & 24.3 & & & & \\
\hline Symptomatic side & & & & & & & & & & & 0.54 \\
\hline Dominant & 17 & 63 & & & & 15 & 71.4 & & & & \\
\hline Non-dominant & 10 & 37 & & & & 6 & 28.6 & & & & \\
\hline $\begin{array}{l}\text { Symptom duration } \\
\text { (months) }\end{array}$ & & & & 4 & $3-60$ & & & & 3 & $3-48$ & 0.51 \\
\hline
\end{tabular}




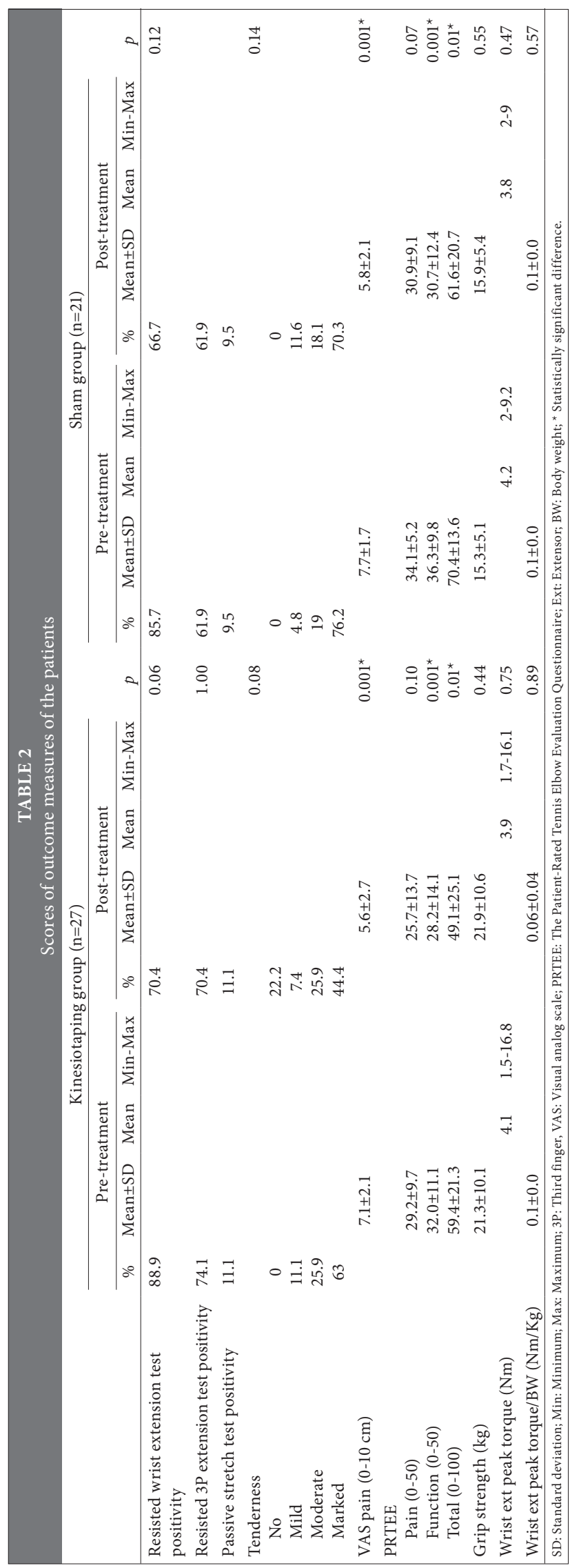

(Biodex Medical Systems Inc., NY, USA). The patients were seated in the testing chair in front of the dynamometer, the forearm in a neutral position supported on a horizontal plane. The joint center of the wrist was aligned with the rotational axis of the dynamometer and the forearm was placed in a pronation position. Testing was performed along a constant range of motion of $110^{\circ}$ on both sides of neutral $\left(-50^{\circ}\right.$ to $\left.+60^{\circ}\right)$. Before the test, all patients performed the wrist extension isokinetic test with three repeats at $60 \% \mathrm{sec}$ as a warm-up and to comprehend the test. Then, wrist flexion and extension concentricconcentric strength measurements were performed with five repeats at $60 \% \mathrm{sec}$. The wrist extension peak torques $(\mathrm{Nm})$ were recorded. The peak torque values were normalized to the body weight (peak torque $/ \mathrm{kg}$ ).

All patients were evaluated before and after the treatment. All assessments were performed by a single researcher who was blinded to the group allocation. None of the patients received any concurrent therapy.

\section{Statistical analysis}

The sample size was calculated based on a previous study. ${ }^{[19]}$ To achieve a $2.1-\mathrm{cm}$ reduction on a $10-\mathrm{cm}$ VAS pain scale, we calculated at least 16 patients per group using 5\% alpha level and $80 \%$ statistical power. Statistical analysis was performed using the PASW version 17.0 software (SPSS Inc., Chicago, IL, USA). Descriptive data were expressed in mean \pm standard deviation (SD), median (min-max) or number and frequency. The Kolmogorov-Smirnov test was used to analyze the distribution of normality. We performed t-tests to compare normally distributed data within the groups and between the groups. Comparison of non-parametric data was analyzed using the Wilcoxon signed-rank test within the groups and using the Mann-Whitney $U$ test between the groups. The chi-square and McNemar tests were used to examine categorical data. A $p$ value of $<0.05$ was considered statistically significant.

\section{RESULTS}

There was no significant difference in the baseline demographic and clinical characteristics of the KT and sham groups $(\mathrm{p}>0.05)$ (Table 1$)$.

The rate of positive resisted wrist extension test and tenderness over the lateral epicondyle were reduced in both groups after the treatment. However, it did not reach statistical significance in either group $(\mathrm{p}>0.05)$. In addition, although not significant $(\mathrm{p}>0.05)$, the rate of positive resisted middle finger test was reduced in 


\begin{tabular}{|c|c|c|c|c|c|}
\hline \multicolumn{6}{|c|}{$\begin{array}{l}\text { TABLE } 3 \\
\text { Change from baseline in outcome measures of the patients }\end{array}$} \\
\hline & \multicolumn{2}{|c|}{ Kinesiotaping group $(\mathrm{n}=27)$} & \multicolumn{2}{|c|}{ Sham group $(n=21)$} & \multirow[b]{2}{*}{$p$} \\
\hline & Mean & Min-Max & Mean & Min-Max & \\
\hline VAS pain & -2 & $-6,-2$ & -2 & $-4,-2$ & 0.42 \\
\hline \multicolumn{6}{|l|}{ PRTEE } \\
\hline Pain & -1 & $-28,-19$ & 2 & $-24,-8$ & 0.92 \\
\hline Function & -4 & $-27.5,-6.5$ & -2 & $-29.5,-8.5$ & 0.38 \\
\hline Total & -7.5 & $-55.5,-23.5$ & -3.5 & $-49.5,-14$ & 0.38 \\
\hline Grip strength & 0.5 & $-5.8,-12.4$ & 0.3 & $-9,-10.9$ & 0.71 \\
\hline Wrist extensor peak torque & -0.1 & $-6.9,-4.6$ & -0.1 & $-43,-1.2$ & 0.79 \\
\hline Wrist extensor peak torque/BW & 0 & $-1.5,-8.3$ & 0 & $-5.4,-2.1$ & 0.61 \\
\hline
\end{tabular}

the KT group after the treatment, while it remained unchanged in the sham group. Passive stretch test positivity did not change after the treatment in both groups. There were no significant differences in the provocative test results and tenderness between the groups ( $>0.05$ ) (Tables 2 and 3 ).

The VAS pain scores significantly decreased after the treatment in both KT and sham groups $(\mathrm{p}<0.05)$. However, there was no statistically difference in the VAS pain scores between the groups $(p>0.05)$ (Tables 2 and 3, Figure 4).

The PRTEE function and total scores also significantly decreased in both groups $(p<0.05)$; however, there was no statistically significant difference between the groups $(p>0.05)$. The PRTEE pain scores remained unchanged in both groups after the treatment and the change between the groups was not statistically significant ( $\mathrm{p}>0.05)$ (Tables 2 and 3 , Figure 5).

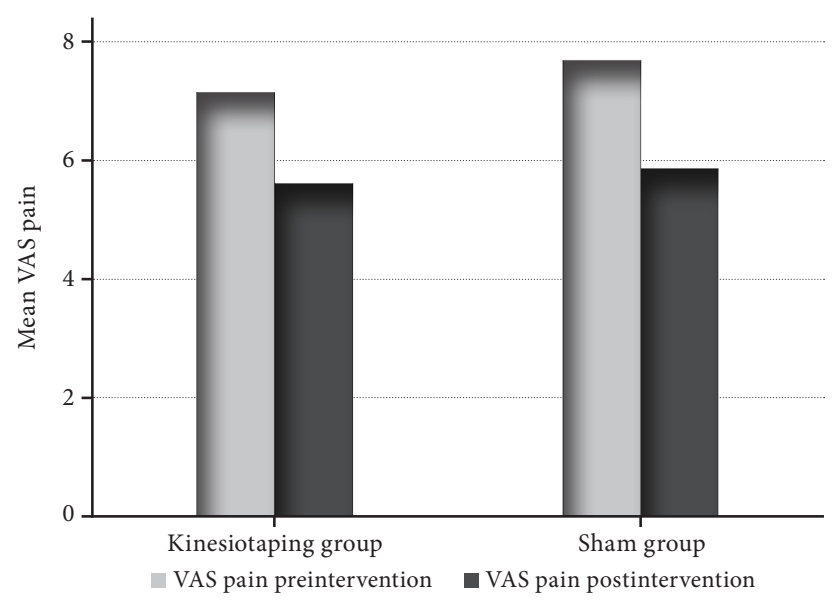

Figure 4. Visual analog scale pain scores of the patients. VAS: Visual analog scale.
The grip strength, wrist extensor peak torque, and peak torque/ $\mathrm{kg}$ values did not significantly change at the end of the treatment in either groups. In addition, there was no significant difference in the grip strength, wrist extensor peak torque, and peak torque $/ \mathrm{kg}$ values between the groups ( $\mathrm{p}>0.05$ ) (Tables 2 and 3 ).

\section{DISCUSSION}

Patients with LE frequently describe pain at or around the lateral epicondyle triggered by the contraction of the wrist extensor muscles. Pain can be mild, moderate or severe and can affect daily living activities and upper extremity functions. Lateral epicondylitis may also cause reduced hand grip strength due to pain. ${ }^{[6]}$ Although there is no standard treatment, conservative therapy should be the first line of treatment. Kinesiotaping has been widely used in the conservative therapy of pain and disability related to musculoskeletal injuries. However, a few

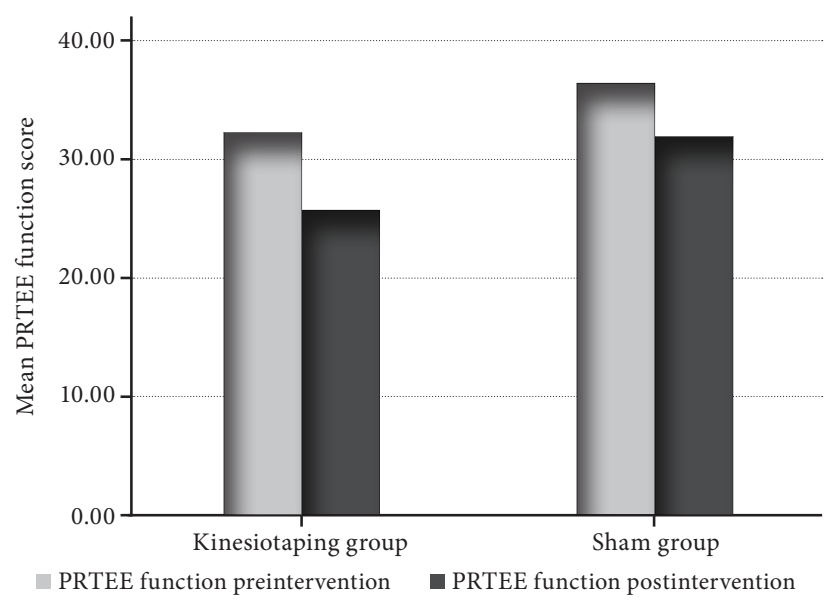

Figure 5. Patient-Rated Tennis Elbow Evaluation Questionnaire function scores of the patients.

PRTEE: Patient-Rated Tennis Elbow Evaluation Questionnaire. 
studies have evaluated the effects of KT on pain, function, grip strength in patients with LE. ${ }^{[13-16,21]}$ In the present study, we evaluated short-term effects of KT on pain, arm function, grip strength, and wrist extensor strength in patients with chronic LE. Our study results showed that pain intensity and functional levels of patients with chronic LE were significantly improved both with KT and sham taping at the end of the treatment period. The most accepted opinion for pain relief provided by $\mathrm{KT}$ is skin tension produced by the pressure of KT. The KT lifts the skin and relieves pressure of mechanoreceptors by generating skin tension. ${ }^{[22]}$ It also increases blood/ lymph circulation due to skin lifting and reduces pain intensity. ${ }^{[23]}$ According to another opinion, $\mathrm{KT}$ increases proprioception through increased stimulation to skin mechanoreceptors and causes pain relief. ${ }^{[15]}$ In our study, we found that both $\mathrm{KT}$ and sham taping decreased pain intensity in patients with chronic LE and there was no significant difference between the groups. Similarly, Cho et al. ${ }^{[21]}$ applied muscle inhibition and fascia correction techniques and found no significant difference in pain relief between the KT and sham groups. In another study, Chang et al. ${ }^{[2]}$ reported that both $\mathrm{KT}$ and placebo taping improved pain condition in athletes suffering from medial epicondylitis. Although the sham application was applied with no tension, it was applied to the same forearm muscles. We consider that sham application decreased pain intensity via proprioceptive theory, providing mechanical stimulation of skin proprioreceptors during the upper extremity activities. The stimulated proprioception increased the patient awareness and adherence to ergonomics and, therefore, pain decreased by applying the sham technique.

In the current study, we evaluated upper extremity functioning with the PRTEE questionnaire which is a more specific to LE than the other questionnaires. We found an improvement in the upper extremity functions in both KT and sham groups. However, we found no statistically significant difference between the groups. Shakeri et al. ${ }^{[15]}$ applied diamond $\mathrm{KT}$ technique and placebo KT (KT without tension) to 15 patients with LE for three times a week and evaluated upper extremity disability using the Disabilities of the Arm, Shoulder and Hand Questionnaire (DASH). The authors found that upper extremity function scores increased significantly in both KT and placebo KT groups. Unlike our results, the aforementioned authors concluded that the KT technique was more effective than the placebo technique on function scores. In another study, Dilek et al. ${ }^{[13]}$ reported that KT (muscle facilitation technique) improved the upper extremity function scores as assessed by the DASH at the end of the treatment in 31 patients with LE; however, there was no control group in this study. It is difficult to compare the results of previous studies with this study due to the differences in KT techniques used, duration of application methods, and measured variables.

In our study, we investigated the effects of KT on strength of the wrist extensor muscles using an isokinetic device in patients with LE. We found no improvement in the wrist extensor muscles strength in either groups at the end of the treatment. No side effects were observed after the application. We also found no significant improvement in the grip strength using a Jamar dynamometer. Similar to our results, a study revealed no significant difference in the hand grip strength between $\mathrm{KT}$ and sham groups using diamond KT technique. ${ }^{[15]}$ Similarly, Cho et al. ${ }^{[21]}$ found no significant differences in the grip strength between the KT group (muscle inhibition and fascia correction techniques) and sham group (without tension). On the contrary, another study demonstrated improvement in the hand grip strength using a Jamar dynamometer with the muscle facilitation KT technique in patients with LE. ${ }^{[13]}$ The discrepancy in the results can be attributed to the different $\mathrm{KT}$ techniques used between the studies.

Nonetheless, there are some limitations to this study. First, the sample size of the study is small which could have reduced the power of the study. Second, we only evaluated the patients before and after the treatment and we were unable to follow the patients at end of the treatment. Therefore, the long-term effects of KT still remain unknown. Finally, there was no untreated placebo group in this study. Despite all these limitations, to the best of our knowledge, this is the first study to evaluate the effects of KT on the wrist extensor strength using an isokinetic device, as previous studies usually used physical examination with a hand dynamometer. The main strength of our study is that a more objective tool was used to detect reduced wrist extensor strength.

In conclusion, our study results suggest that both $\mathrm{KT}$ and sham taping provide similar improvement in pain relief through arm functions in patients with chronic LE. However, further studies with a larger sample size and longer follow-up period are needed to confirm the effects of KT in patients with chronic LE. 


\section{Declaration of conflicting interests}

The authors declared no conflicts of interest with respect to the authorship and/or publication of this article.

\section{Funding}

The authors received no financial support for the research and/or authorship of this article.

\section{REFERENCES}

1. Nourbakhsh MR, Fearon FJ. The effect of oscillating-energy manual therapy on lateral epicondylitis: a randomized, placebo-control, double-blinded study. J Hand Ther 2008;21:4-13.

2. Smidt $\mathrm{N}$, van der Windt DA. Tennis elbow in primary care. BMJ 2006;333:927-8.

3. Vicenzino B, Cleland JA, Bisset L. Joint manipulation in the management of lateral epicondylalgia: a clinical commentary. J Man Manip Ther 2007;15:50-6.

4. Pienimäki $T$, Tarvainen TK, Siira $P$, Vanharanta $H$. Progressive strengthening and stretching exercises and ultrasound for chronic lateral epicondylitis. Physiotherapy 1996;82:522-30.

5. Stasinopoulos D, Johnson MI. Cyriax physiotherapy for tennis elbow/lateral epicondylitis. Br J Sports Med 2004;38:675-7.

6. Ahmad Z, Siddiqui N, Malik SS, Abdus-Samee M, Tytherleigh-Strong G, Rushton N. Lateral epicondylitis: a review of pathology and management. Bone Joint J 2013;95B:1158-64.

7. Aam BS, Nejad L. Systematic review: effects of using Kinesio tape on treatment of lateral epicondylitis. Phys Treat. 2014;4(3):115-122.

8. Wu WT, Hong CZ, Chou LW. The kinesio taping method for myofascial pain control. Evid Based Complement Alternat Med 2015;2015:1-9.

9. Chol JH, Yoo KT, An HJ, Chol WS, Koo JP, Kim JI, Kim NJ. The effects of taping, stretching, and joint exercise on hip joint flexibility and range of motion. J Phys Ther Sci 2016;28:1665-1668.

10. Shamsoddini A, Hollisaz MT, Hafezi R. Initial effect of taping technique on wrist extension and grip strength and pain of Individuals with lateral epicondylitis. Iran Rehabil J 2010;8:24-28.

11. Kachanathu SH. Forearm band versus elbow taping: as a management of lateral epicondylitis. J Muscoskel Res 2013;16:1-9.

12. Prabhakar AJ, Kage V. Comparison of Cyriax physiotherapy and taping technique in subjects with tenis elbow: a randomized clinical trial. Rom J Physical Ther 2013;19:41-8.

13. Dilek B, Batmaz I, Sarıyıldız MA, Sahin E, Ilter L, Gulbahar $\mathrm{S}$, et al. Kinesio taping in patients with lateral epicondylitis. J Back Musculoskelet Rehabil 2016;29:853-8.

14. Schneider M, Rhea M, Bay C. The effect of Kinesio Tex tape on muscular strength of the forearm extensors on collegiate tenis athletes. Kinesio Taping Association International Published Research; 2010.

15. Shakeri H, Soleimanifar M, Arab AM, Hamneshin Behbahani S. The effects of KinesioTape on the treatment of lateral epicondylitis. J Hand Ther 2018;31:35-41.

16. Jesensek Papez B. The impact of kinesio taping on pain relief in patients with lateral epicondylitis. ACTA MedicoBiotechnica 2014;7:45-52.

17. Artioli DP, Bertolini GRF. Kinesio taping: application and results on pain: systematic review. Fisioter Pesq 2014;21:94-9.

18. Gramatikova M, Nikolova E, Mitova S. Nature, application and effect of Kinesio - taping. APES 2014;4:115-9.

19. Cho YT, Hsu WY, Lin LF, Lin YN. Kinesio taping reduces elbow pain during resisted wrist extension in patients with chronic lateral epicondylitis: a randomized, double-blinded, cross-over study. BMC Musculoskelet Disord 2018;19:193.

20. Altan L, Ercan I, Konur S. Reliability and validity of Turkish version of the patient rated tennis elbow evaluation. Rheumatol Int 2010;30:1049-54.

21. Cho YT, Hsu WY, Lin LF, Lin YN. Kinesio taping reduces elbow pain during resisted wrist extension in patients with chronic lateral epicondylitis: a randomized, double-blinded, cross-over study. BMC Musculoskelet Disord 2018;19:193.

22. Yang JM, Lee JH. Is Kinesio Taping to Generate Skin Convolutions Effective for Increasing Local Blood Circulation? Med Sci Monit 2018;24:288-93.

23. Malicka I, Rosseger A, Hanuszkiewicz J, Woźniewski M. Kinesiology Taping reduces lymphedema of the upper extremity in women after breast cancer treatment: a pilot study. Prz Menopauzalny 2014;13:221-6.

24. Chang HY, Wang CH, Chou KY, Cheng SC. Could forearm Kinesio Taping improve strength, force sense, and pain in baseball pitchers with medial epicondylitis? Clin J Sport Med 2012;22:327-33. 